\title{
A novel technique for tumor localization and targeted lymphatic mapping in early-stage lung cancer
}

\author{
Krista J. Hachey, MD, ${ }^{a}$ Christopher S. Digesu, MD, ${ }^{\text {a }}$ Katherine W. Armstrong, MPH, ${ }^{a}$ \\ Denis M. Gilmore, MD, ${ }^{\mathrm{a}, \mathrm{b}}$ Onkar V. Khullar, MD,${ }^{\mathrm{a}, \mathrm{c}}$ Brian Whang, MD, ${ }^{\mathrm{a}}$ Hisashi Tsukada, MD, PhD, ${ }^{\mathrm{a}}$ and \\ Yolonda L. Colson, $\mathrm{MD}, \mathrm{PhD}^{\mathrm{a}}$
}

\begin{abstract}
Objective: To investigate safety and feasibility of navigational bronchoscopy (NB)-guided near-infrared (NIR) localization of small, ill-defined lung lesions and sentinel lymph nodes (SLN) for accurate staging in patients with non-small cell lung cancer (NSCLC).

Methods: Patients with known or suspected stage I NSCLC were enrolled in a prospective pilot trial for lesion localization and SLN mapping via NB-guided NIR marking. Successful localization, SLN detection rates, histopathologic status of SLN versus overall nodes, and concordance to initial clinical stage were measured. Ex vivo confirmation of $\mathrm{NIR}^{+}$SLNs and adverse events were recorded.

Results: Twelve patients underwent NB-guided marking with indocyanine green of lung lesions ranging in size from 0.4 to $2.2 \mathrm{~cm}$ and located 0.1 to $3 \mathrm{~cm}$ from the pleural surface. An NIR " tattoo" was identified in all cases. Ten patients were diagnosed with NSCLC and 9 SLNs were identified in 8 of the 10 patients, resulting in an $80 \%$ SLN detection rate. SLN pathologic status was $100 \%$ sensitive and specific for overall nodal status with no false-negative results. Despite previous nodal sampling, one patient was found to have metastatic disease in the SLN alone, a $12.5 \%$ rate of disease upstaging with NIR SLN mapping. SLN were detectable for up to 3 hours, allowing time for obtaining a tissue diagnosis and surgical resection. There were no adverse events associated with NB-labeling or indocyanine green dye itself.
\end{abstract}

Conclusions: NB-guided NIR lesion localization and SLN identification was safe and feasible. This minimally invasive image-guided technique may permit the accurate localization and nodal staging of early stage lung cancers. (J Thorac Cardiovasc Surg 2017;154:1110-8)

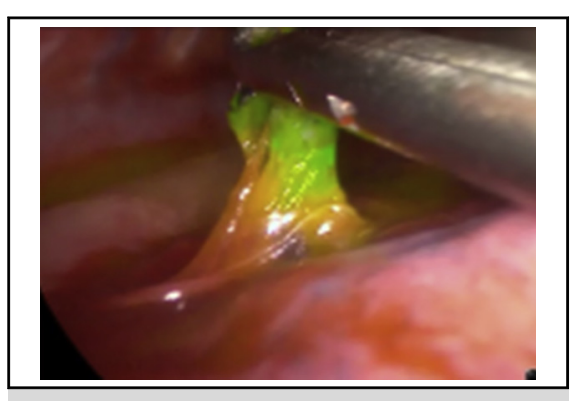

Level 7 near-infrared positive sentinel lymph node after navigational bronchoscopy-guided perilesional "tattoo" with indocyanine green.

Central Message

Navigational bronchoscopy-guided near-infrared tumor marking is feasible and allows localization and nodal staging of early stage lung cancers.

Perspective

Two major barriers to curative treatment of early stage lung cancer are surgical: (1) challenge in identifying small, ill-defined lesions intraoperatively for resection and (2) inadequate sampling and histologic scrutiny of tumor-associated lymph nodes. Navigational bronchoscopy-guided near-infrared marking is a novel technique that permits tumor localization and targeted staging of early lung cancers.

See Editorial Commentary page 1119.

See Editorial page 1077.
From the a'Division of Thoracic Surgery, Brigham and Women's Hospital, Boston, Mass; ${ }^{b}$ Department of Thoracic Surgery, Vanderbilt University Medical Center, Nashville, Tenn; and ${ }^{\mathrm{c}}$ Division of Cardiothoracic Surgery, Emory University School of Medicine, Atlanta, Ga.

This work was supported by National Institutes of Health R01CA131044, the Karl Storz Society of University Surgeons Resident Scholar Award (K.H.), and the Brigham and Women's Hospital Advanced Training in Surgical Oncology T32 Fellowship (5T32CA009535) for K.H., C.D., D.G., and O.K.

Drs K.J.H. and C.S.D. contributed equally to this work.

Received for publication June 2, 2016; revisions received Dec 1, 2016; accepted for publication Dec 7, 2016; available ahead of print March 6, 2017.

Address for reprints: Yolonda L. Colson, MD, PhD, Division of Thoracic Surgery, Department of Surgery, Brigham and Women's Hospital, 15 Francis St, Boston, MA 02115 (E-mail: ycolson@ @artners.org).

$0022-5223 / \$ 36.00$

Copyright $(\odot 2017$ by The American Association for Thoracic Surgery

http://dx.doi.org/10.1016/j.jtcvs.2016.12.058
Lung cancer is the leading cause of cancer-related mortality in the United States, with an estimated 158,080 deaths expected to occur in 2016. ${ }^{1}$ Despite "curative" resection and lymphadenectomy (LAD), there is a $30 \%$ recurrence rate and a $60 \%-80 \% 5$-year survival in early stage non-small cell

Scanning this QR code will take you to a supplemental video for the article. 

Abbreviations and Acronyms
CT = computed tomography
GGO = ground-glass opacity
HSA = human serum albumin
ICG = indocyanine green
LAD = lymphadenectomy
NB = navigational bronchoscopy
NIR = near-infrared
NSCLC $=$ non-small cell lung cancer
PET = positron emission tomography
SLN $=$ sentinel lymph node

lung cancer (NSCLC). ${ }^{2}$ Attempts to improve these outcomes have led to the initiation of national lung cancer screening, where an estimated 2 million Americans will be diagnosed with a new pulmonary nodule, and more than 80,000 will require surgery for diagnosis and treatment. ${ }^{3-5}$

Unfortunately, many very early cancers discovered with screening are too small or ill-defined, as with ground-glass opacities (GGO), preventing localization for limited surgical resection or biopsy. Therefore, these small lesions are followed with imaging surveillance until they are large enough for diagnostic percutaneous biopsy or amenable to intraoperative localization. The concern of the surgeon and patient is that disease progression can occur during surveillance, thus potentially undermining the benefit of early detection. If a small or ill-defined lesion can be localized intraoperatively, limited surgical resection may not only offer a diagnosis but is also potentially curative for some of these small, peripheral lesions, if nodal disease is in fact absent. ${ }^{6-8}$ Furthermore, many patients with poor cardiopulmonary reserve may only be candidates for limited resection, and thus precise intraoperative localization for lung parenchyma preservation with a negative surgical resection margin is critical for maximizing long-term outcomes.

Despite negative preoperative imaging to rule out occult locoregional lymph node metastases, pathologic upstaging found at the time of surgical resection occurs in up to $18 \%$ of patients. ${ }^{9,10}$ Furthermore, nodal staging is inadequate in more than $50 \%$ of limited resections performed for early-stage cancers, and standard hematoxylin and eosin analysis of all lymph nodes in the specimen identifies occult metastases 16\%-18\% less often than when individual nodes are scrutinized histologically. ${ }^{11,12}$ Occult nodal disease leads to understaging and missed opportunities for adjuvant therapy and may result in poor clinical outcomes. ${ }^{13-17}$ Thus, 2 major barriers facing the curative treatment of early-stage lung cancer are surgical: the current challenge in identifying small, ill-defined lesions intraoperatively for parenchymasparing resections and the inadequate sampling and histologic scrutiny of tumor-associated lymph nodes.
Our previous phase I dose-titration trial using transpleural, peritumoral injection of the Food and Drug Administrationapproved near-infrared (NIR) dye indocyanine green (ICG) in 41 patients demonstrated no adverse events, excellent real-time imaging via minimally invasive NIR thoracoscopy, and a dose-dependent increase in NIR signal within sentinel lymph nodes (SLNs). ${ }^{18}$ Although spillage of dye can occur after transpleural injection, SLNs were identified in $100 \%$ of patients at the optimized ICG dose. Importantly, transpleural injection required intraoperative palpation of the lesion for peritumoral injection, limiting this technique to superficial and/or relatively large lesions.

We conducted the first-in-human pilot trial of patients with suspected or known early-stage NSCLC undergoing intraoperative navigational bronchoscopy (NB)-guided transbronchial, peritumoral ICG injection, subsequent NIR lesion localization, resection, and SLN mapping via minimally invasive thoracoscopic surgery. We aimed to show this comprehensive image-guided technique is both safe and feasible for both localization of early-stage lung cancers and targeting of tumor-associated SLNs.

\section{METHODS}

\section{Study Design and Patients}

This prospective pilot trial evaluates the feasibility of NB-guided peritumoral ICG injection for NIR "tattoo" marking of suspected or known lung cancers and $\mathrm{NIR}^{+}$SLN retrieval for nodal staging (Figure 1). The trial protocol was approved by the Partners Internal Review Board (Boston, Mass), and all cases were conducted at Brigham and Women's Hospital (Boston, Mass). Primary endpoints included visualization of the NIR " tattoo," complete resection of the lesion, identification of $\mathrm{NIR}^{+}$SLNs, and histopathologic status of SLNs versus overall LNs in the LAD specimen. In addition, adverse events associated with NB-labeling or to ICG dye itself were recorded.

Twelve patients with clinically suspected or biopsy-proven T1N0 NSCLC scheduled to undergo minimally invasive lung resection were enrolled between March and December 2015 following informed consent for surgery and independently for the study protocol. Exclusion criteria included age $<18$ years old, pregnancy or breastfeeding, history of iodide or seafood allergy, suspected or known metastatic nodal disease, and previous neoadjuvant chemotherapy or radiation therapy to the lung. Although lobectomy is the standard of care for lung cancer, initial patients selected for this trial were those undergoing minimally invasive wedge resection for diagnosis or treatment either due to patient comorbidity, lesion characteristics, ${ }^{6}$ or patient preference as the aim of this trial was to test our technique for both lesion localization and targeted lymphatic mapping. All study patients underwent standard preoperative chest computed tomography (CT) \pm positron emission tomography (PET)/CT for staging. Four patients with enlarged or suspicious lymph nodes had documented negative nodal pathology via cervical mediastinoscopy or endobronchial ultrasound prior to lung resection. All patients found to have NSCLC underwent LAD at the time of surgery.

\section{NB-Guided ICG Peritumoral Injection}

$\mathrm{NB}$ is a real-time localization system that uses 3-dimensional images generated from a preoperative NB-protocol chest CT or PET/CT scan with $1 \times 0.8-\mathrm{mm}$ slice intervals. The superDimension system(Covidien, Minneapolis, Minn) used creates a "road map" through the airway anatomy to guide a NB catheter to the lesion of interest for intraoperative biopsy and peritumoral ICG injection. ICG (Novadaq Technologies, Bonita Springs, 


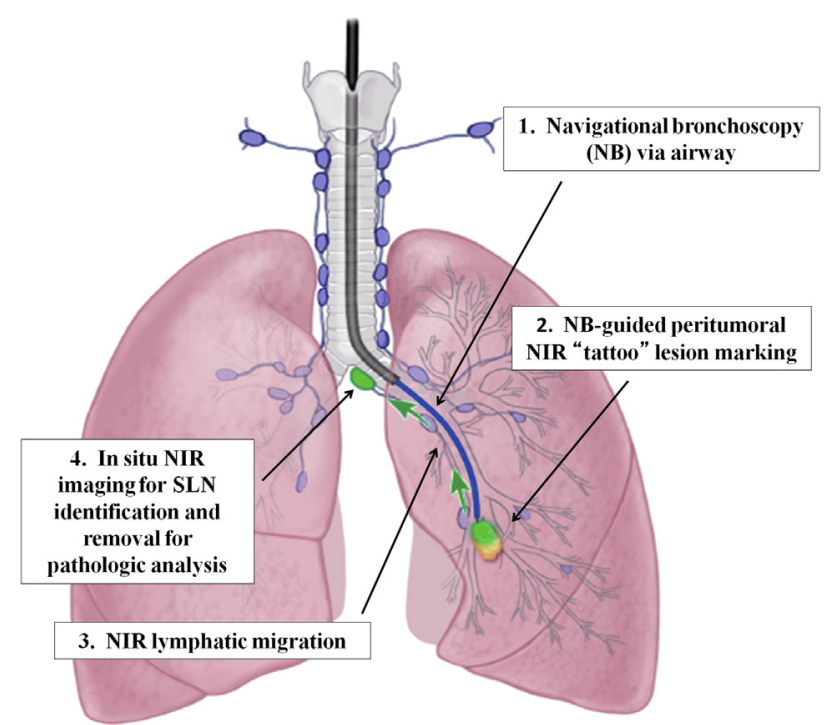

FIGURE 1. NB-guided NIR imaging technique. NB-guided lesion localization for $\mathrm{NIR}^{+}$"tattoo," NIR lymphatic migration, and in situ $\mathrm{NIR}^{+}$SLN identification for in-depth pathologic analysis. NB, Navigational bronchoscopy; NIR, near-infrared; $S L N$, sentinel lymph node.

Fla) was diluted to $2.5 \mathrm{mg} / \mathrm{mL}$ using $25 \%$ human serum albumin (ICG:HSA), as albumin has been shown to increase the effective hydrodynamic diameter of ICG almost 6-fold, resulting in increased lymphatic retention. ${ }^{19}$ Based on our group's previous transpleural NIR dose escalation trial for lung cancer, ${ }^{15}$ a dose of 1.25-2.5 mg (0.5-1 mL) ICG was used. Using the NB catheter location registration, access to within a centimeter of the lesion was obtained via the airway. A needle was then advanced towards the lesion to inject ICG just deep to the lesion of interest. This allowed ICG marking of the deep margin of the lesion, ensuring the entire lesion was removed with resection of the ICG labeled parenchyma. The number of peritumoral injections used in each case ranged from 1 to 3 discrete injections, depending on lesion location, feasibility of multiple injection sites, and the number of lesions to be localized. The "tattoo" was visualized from the pleural surface due to the property of NIR light to penetrate tissue. ${ }^{20} \mathrm{NB}$-guided lesion biopsy with fluoroscopy was performed at the discretion of the operating surgeon.

\section{NIR Image-Guided Resection and Lymphatic Mapping}

After NB-guided peritumoral injection, patients were repositioned to the appropriate lateral decubitus position for resection via video-assisted thoracoscopic surgery. Real-time NIR fluorescence images were obtained intraoperatively using a minimally invasive NIR PINPOINT imaging system (Novadaq) including a 10-mm, 30-degree NIR thoracoscopic camera. ICG signal was detected in vivo at the site of peritumoral "tattoo" in all patients, with minimal NIR autofluorescence or background signal from normal human tissue. All patients underwent lung resection, and those with confirmed NSCLC underwent lymph node sampling comprising the LAD specimen with NIR imaging for SLN identification and resection. Specimens were imaged ex vivo to confirm NIR signal. The decision for definitive wedge resection versus lobectomy was based on lesion characteristics (identification of benign disease, multifocal adenocarcinoma, or metastatic disease), limited pulmonary status, or patient preference. For diagnoses other than NSCLC (benign lesions or metastases from another primary site), LAD and NIR SLN imaging were not performed.

\section{Pathologic Analysis}

The surgical specimen and the individually labeled nodal specimens, including $\mathrm{NIR}^{+}$SLNs, were sent for routine histopathologic analysis. All LNs were fixed in formalin and embedded in paraffin for hematoxylin and eosin staining. Pathologic status was determined by an experienced lung pathologist without knowledge of which node was identified as $\mathrm{NIR}^{+}$intraoperatively.

\section{Statistical Analysis}

The data reported is descriptive of the events that occurred in the trial with mean and standard deviation reported where appropriate. Specificity and sensitivity of the SLN are calculated based on the pathologic status of the $\mathrm{NIR}^{+}$SLN in comparison with all nodes sampled along with the LAD.

\section{RESULTS \\ Patient and Lesion Characteristics}

Twelve patients, 10 female and 2 male, underwent NB-guided NIR marking of lung lesions. Mean patient age was 62 years old with a standard deviation of 6.7 years. A total of 15 lung lesions, all malignant, were resected ranging in size from 0.4 to $2.2 \mathrm{~cm}$, with a depth from the pleural surface of 0.1 to $3.0 \mathrm{~cm}$ (Table 1). Seven lesions were characterized as solid on preoperative imaging, 6 were semisolid, 1 was a pure GGO, and 1 was not evident on preoperative imaging. Lesions were identified in the right upper lobe $(n=4)$, right lower lobe $(n=2)$, left upper lobe $(n=6)$, and left lower lobe $(n=3)$. Wedge resection was performed for all lesions to establish a pathologic diagnosis with completion lobectomy in 1 case. Two patients were diagnosed with metastatic disease from another primary and 10 patients with NSCLC.

\section{NB-Guided NIR Lesion Localization and Resection}

NB-guided NIR "tattoo" marking was performed under general anesthesia with the patient supine and immediately before lung resection in all 12 cases with all 14 known lesions NIR marked and intraoperatively identified. The size of the visualized pleural "tattoo" depended on the depth and location of injection of ICG. Two separate lesions were marked with $0.5 \mathrm{~mL}$ of ICG:HSA per lesion in patients 5 and 12. These patients underwent wedge resections for diagnosis of potential multifocal disease. Patient 12 was discovered on final pathology to have a third focus of adenocarcinoma not palpable or identified on preoperative imaging, but it was removed within the wedge resection of an adjacent NIR marked tumor.

Total NB time for peritumoral injection, which included biopsy and on-site cytologic interpretation as requested by the surgeon in 7 cases, averaged 34.5 minutes with approximately 20 minutes being for the entire navigation, dye preparation, and lesion "tattoo" if biopsy was not required. Total fluoroscopy time for injection of ICG alone ranged from 3 to 20 seconds and for patients who also 
TABLE 1. Characteristics of pulmonary nodules and NB-guided NIR "tattoo" marking

\begin{tabular}{|c|c|c|c|c|c|c|c|c|c|}
\hline \multirow{2}{*}{$\begin{array}{c}\text { Patient } \\
\text { no. }\end{array}$} & \multirow{2}{*}{$\begin{array}{c}\text { Lesion } \\
\text { location }\end{array}$} & \multirow{2}{*}{$\begin{array}{c}\text { Final pathologic } \\
\text { size, } \mathrm{cm}\end{array}$} & \multirow{2}{*}{ Lesion type } & \multirow{2}{*}{$\begin{array}{c}\text { Depth from } \\
\text { pleural } \\
\text { surface, } \mathbf{c m}^{*}\end{array}$} & \multicolumn{2}{|c|}{$\begin{array}{l}\text { Fluoro time for } \\
\text { ICG injection, s }\end{array}$} & \multirow{2}{*}{$\begin{array}{c}\text { Time from } \\
\text { ICG injection } \\
\text { to NIR tattoo } \\
\text { ID, min } \\
\end{array}$} & \multirow{2}{*}{$\begin{array}{c}\text { Margin } \\
\text { distance of } \\
\text { initial wedge } \\
\text { resection, } \mathbf{c m} \dagger\end{array}$} & \multirow{2}{*}{ Lesion diagnosis } \\
\hline & & & & & ICG alone & Total & & & \\
\hline 1 & RLL & 1.8 & Solid & 1.0 & $\mathrm{NR} \ddagger$ & 19.7 & 65 & 1.2 & Adenocarcinoma \\
\hline 2 & LLL & 1.9 & Solid & 2.0 & NR & 26 & 60 & $0.1 \S$ & Adenocarcinoma \\
\hline 3 & LLL & 2.0 & Solid & 0.5 & NR & 25 & 52 & N/A & $\begin{array}{l}\text { Atypical spindle cel } \\
\text { neoplasm }\end{array}$ \\
\hline 4 & LUL & 1.3 & Mixed GGO/solid & 1.0 & - & - & 38 & 0.7 & Adenocarcinoma \\
\hline \multirow[t]{2}{*}{$5 \pi$} & RUL & 1.6 & Solid & 3.0 & 20 & 33 & 55 & 0.5 & Metastatic \\
\hline & RUL & 0.4 & Solid & 0.8 & & & 50 & 0.4 & leiomyosarcoma \\
\hline 6 & LUL & 1.5 & Solid & 2.1 & 18 & 18 & $161 \|$ & 1.5 & Adenocarcinoma \\
\hline 7 & LLL & 0.7 & Mixed GGO/solid & 0.1 & 14 & 17 & 43 & 1.0 & Adenocarcinoma \\
\hline 8 & LUL & 1.3 & Mixed GGO/solid & 0.4 & - & - & 43 & 0.2 & Adenocarcinoma \\
\hline 9 & LUL & 2.2 & Solid & 0.1 & 6 & 14 & 66 & 1.8 & $\begin{array}{l}\text { Squamous cell } \\
\text { carcinoma }\end{array}$ \\
\hline 10 & LUL & 0.9 & Mixed GGO/solid & 0.1 & 12 & 12 & 48 & 1.1 & Adenocarcinoma \\
\hline 11 & LUL & 1.1 & Mixed GGO/solid & 1.6 & 3 & 10 & 73 & 2.8 & Adenocarcinoma \\
\hline \multirow[t]{2}{*}{12 ब } & RUL & 2.1 & Mixed GGO/solid & 0.3 & - & - & 64 & 2.4 & Adenocarcinoma \\
\hline & RLL & 1.5 & GGO & 1.0 & & & 81 & 0.1 & \\
\hline
\end{tabular}

$\overline{N B}$, Navigational bronchoscopy; $N I R$, near-infrared; $I C G$, indocyanine green; $R L L$, right lower lobe; $N R$, not reported; $L L L$, left lower lobe; $N / A$, not available; $L U L$, left upper

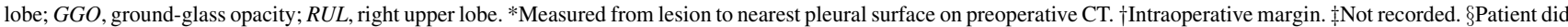
undergo completion lobectomy. "Case included needle localization under CT guidance after NIR "tattoo" and before conversion to VATS. ๆTwo separate lesions were NIR marked with $1.25 \mathrm{mg}$ of ICG (in $0.5 \mathrm{~mL}$ of ICG:HSA) per lesion. Patient 12 also had an incidentally found third focus of adenocarcinoma on final pathology.

underwent NB-biopsy ranged from 19.7 to 26 seconds with a total dose of 0.93-3.94 mGy.

Immediately after placement of the NIR thoracoscope, the NIR "tattoo" was identified successfully on the pleural surface corresponding to each lesion in all cases. The average time to visualization after injection was 65 minutes with a standard deviation of 31 minutes. Given the depth of the lesion in patient 6 , needle localization under CTguidance after ICG injection was unsuccessfully attempted as a backup strategy before video-assisted thoracoscopic surgery delaying the assessment of NIR marking which was still readily visible at 161 minutes (Figure 2). For each patient, the site of $\mathrm{NIR}^{+}$"tattoo" colocalized with the primary nodule identified on all frozen section analyses and was associated with negative surgical margins in all cases (mean margin distance $0.9 \mathrm{~cm} \pm 0.7 \mathrm{~cm}$ ). The average lesion to lesion:margin ratio was $1.3 \pm 0.8$. No clinically significant adverse events after $\mathrm{NB}$ and the injection of ICG were noted including bleeding, pneumothoraces, or bronchospasm.

\section{NB-Guided NIR Imaging for SLN Identification}

Ten of the 12 patients were found to have primary lung cancer for which SLN mapping and subsequent LAD were performed. SLN mapping was not performed in 2 patients without primary lung cancer. At least one $\mathrm{NIR}^{+} \mathrm{SLN}$ was identified in 8 of the 10 lung cancer cases, for an $80 \%$ detection rate (Table 2). Notably, $75 \%$ of SLNs identified were in N2 stations increasing the likelihood of finding disease that can upstage patients to stage III, as occurred in patient 1 . SLNs were not identified in patients 6 and 8 . As mentioned, patient 6 was delayed due to an unsuccessful attempt at needle localization. Although the NIR "tattoo" readily aided in tumor localization, the delay resulted in greater than 3 hours elapsing between the time of injection and examination of lymph node stations. Patient 8 also had a visible $\mathrm{NIR}^{+}$"tattoo" of a peripheral lesion, but ICG was noted to have extravasated through the visceral pleura, resulting in a smaller actual dose delivered to the lung parenchyma and no $\mathrm{NIR}^{+}$SLN was identified. In addition, this patient had a diagnosis of sarcoidosis with significant fibrosis involving the lymph nodes and associated lung parenchyma which may have also contributed to this failure. Patient 7 underwent concurrent methylene blue injection of the lesion, which was visible at the site of the $\mathrm{NIR}^{+}$"tattoo"; however the SLN was only visible with ICG and no methylene blue was present within the LAD specimen. Representative cases in which a SLN was identified are shown in Figure 3 and Video 1.

\section{Pathologic Analysis}

Both the lung and SLN specimens were imaged ex vivo before pathologic analysis to confirm $\mathrm{NIR}^{+}$status. Pathologic analysis of resected lesions revealed primary lung adenocarcinoma $(n=9)$, squamous cell carcinoma $(\mathrm{n}=1)$, atypical spindle cell neoplasm $(\mathrm{n}=1)$, and metastatic leiomyosarcoma $(\mathrm{n}=1)$. In all cases in which LAD was performed, a negative SLN was reflective of the status of all other nodes in the LAD specimen. Importantly, the $\mathrm{NIR}^{+}$level 7 SLN identified in patient 1 was the only 



FIGURE 2. NB-guided NIR "tattoo" of lesion $2.1 \mathrm{~cm}$ from the pleural surface. A, Navigational bronchoscopy guides the injection of $1 \mathrm{~mL}$ of ICG. B, Axial CT image demonstrating a 1.1-cm lesion (yellow marker) that is $2.1 \mathrm{~cm}$ from the pleural surface (blue arrows). C, NIR "tattoo" is easily identifiable from the pleural surface nearly 4 hours after ICG injection.

pathologic positive lymph node identified within the LAD specimen, resulting in upstaging from a negative nodal status (despite preoperative cervical mediastinoscopy) to stage IIIa disease. On the basis of these findings, the pathologic status of the 9 identified SLN was $100 \%$ sensitive and specific for overall nodal disease.

\section{DISCUSSION}

This is the first reported human pilot trial to demonstrate the safety and feasibility of NB-guided peritumoral NIR marking of lung lesions and mapping of tumor-associated SLNs. Twelve patients successfully underwent NB-guided marking, allowing for lung lesion localization and resection with a negative margin in all patients. SLNs were identified in $80 \%$ of the 10 patients found to have NSCLC. This minimally invasive technique allows for the accurate localization and staging of small, ill-defined pulmonary nodules and GGOs representing a significant advancement in the way we manage, treat, and stage early lung cancers.

TABLE 2. SLN yield and specimen pathology

\begin{tabular}{|c|c|c|c|c|c|c|}
\hline $\begin{array}{c}\text { Patient } \\
\text { no. }\end{array}$ & $\begin{array}{c}\text { SLN station } \\
\text { (no. nodes) }\end{array}$ & $\begin{array}{l}\text { Time to SLN } \\
\text { resection (min) } \\
\text { (from injection) }\end{array}$ & SLN pathology & Other lymph node stations (no. nodes)* & $\begin{array}{c}\text { Other lymph } \\
\text { node pathology }\end{array}$ & $\begin{array}{l}\text { Pathologic } \\
\text { stage }\end{array}$ \\
\hline 1 & Level 7 (1) & 124 & Positive & $\begin{array}{l}\text { Level } 4 \text { (1); Level } 7 \text { (1); Level } 10 \text { (1); } \\
\quad \text { Level } 11 \text { (5) }\end{array}$ & Negative & T1aN2 \\
\hline 2 & Level 10 (1) & 180 & Negative & Level 5 (1); Level 10 (4); other hilar (6) & Negative & T1aN0 \\
\hline 4 & Level 5 (1) & 155 & Negative & Level 11 (2); Level 7 (1) & Negative & T1aN0 \\
\hline 6 & - & - & - & Level 5 (2); Level 7 (5) & Negative & T1aN0 \\
\hline 7 & Level 7 (3) & 64 & Negative & Level $9(3)$ & Negative & T1aN0 \\
\hline 8 & - & - & - & Level 5 (1); Level 7 (1); Level 10L (1) & Negative & T1aN0 \\
\hline 9 & Level 5 (1) & 134 & Negative & Level 10L (1) & Negative & T1bN0 \\
\hline 10 & Level 7 (1) & 114 & Negative & Level 5 (1); Level 10 (1) & Negative & T1aN0 \\
\hline 11 & Level 5 (1); Level 7 (1) & 102 & Negative & - & Negative & T1aN0 \\
\hline 12 & Level 11R (1) & 92 & Negative & Level 4R (1); Level 7 (1) & Negative & $\begin{array}{l}\text { T1bN0 } \\
\text { T1aN0 } \\
\text { T1aN0 }\end{array}$ \\
\hline
\end{tabular}

SLN, Sentinel lymph node. *Additional nodes removed as part of the routine lymphadenectomy. 


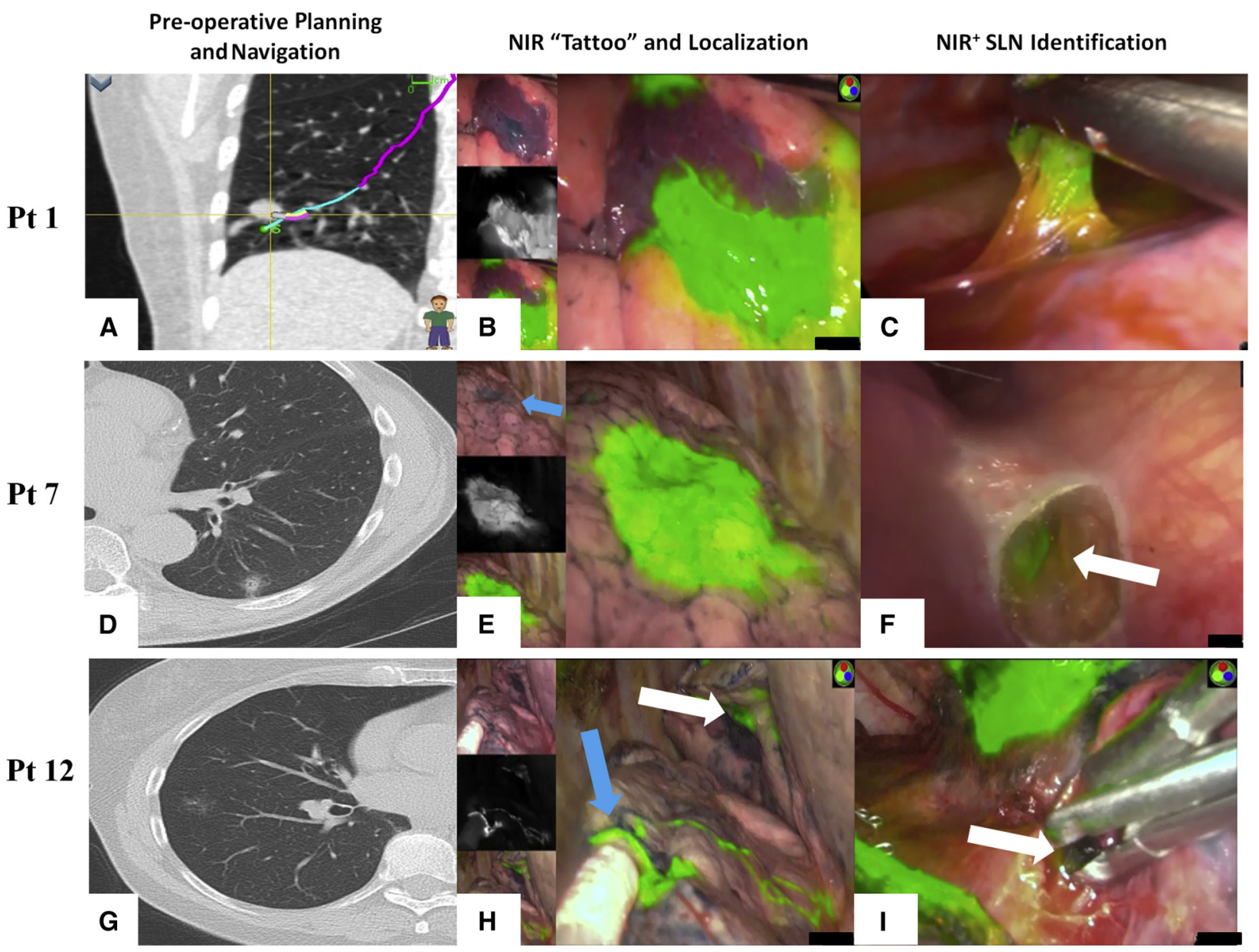

FIGURE 3. NB-guided NIR "tattoo" and SLN identification. A, NB-guided lesion localization for transbronchial, peritumoral ICG injection. B, $\mathrm{NIR}^{+}$"tattoo" on pleural surface $\sim 1$ hour after ICG injection (3 injections used). C, In vivo NIR ${ }^{+}$level 7 SLN identification $\sim 2$ hours after ICG injection. D, Axial CT of a 1.2-cm LLL lesion. E, NIR ${ }^{+}$ICG "tattoo" is nearly identical to a concomitant methylene blue marking (blue arrow). F, In vivo NIR ${ }^{+}$level 7 SLN (white arrow) identified in situ by ICG alone with no methylene blue. G, Axial CT of 1.5-cm ill-defined, pure groundglass opacity. H, In vivo NIR lymphatic track from RLL peritumoral "tattoo" (blue arrow) to level 11R nodal station, RUL peritumoral "tattoo" (white arrow). I, In vivo $\mathrm{NIR}^{+}$level 11R SLN (white arrow). NB, Navigational bronchoscopy; NIR, near-infrared; SLN, sentinel lymph node.

Nodal status is a significant prognostic indicator in lung cancer, yet many patients still do not undergo proper nodal staging leading to potentially understaged and undertreated disease, which may contribute to poor overall survival and relatively high recurrence rates. ${ }^{2,12-17}$ Although preoperative imaging, in particular $\mathrm{CT}$ and $\mathrm{PET} / \mathrm{CT}$, is necessary for clinical staging, histologic diagnosis is still required to ensure N0 status, given the relatively low sensitivity and specificity for detecting metastatic nodal disease in early-stage lung cancers. ${ }^{21,22}$ The high falsenegative rate is evident by the nearly $18 \%$ incidence of pathologic upstaging reported in early-stage lung cancers, and, in fact, our study resulted in the upstaging to stage III disease in $12.5 \%(1 / 8)$ of patients in whom SLNs were analyzed. ${ }^{9,10}$

Although nodal sampling has been shown to be equivalent to radical LAD, SLN mapping offers distinct advantages including targeting the tumor-draining node(s) that are at increased risk of harboring metastatic disease and also permitting focused in-depth histologic scrutiny. ${ }^{23}$ In addition, patients with early-stage NSCLC are prone to developing a second primary NSCLC at a rate of $1 \%-2 \% / y e a r$. In the future, targeted nodal assessment could potentially allow for subsequent nodal staging of a second ipsilateral lung cancer, an opportunity that is currently not possible if a full LAD has previously been performed. ${ }^{24}$

Under the current NCCN guidelines, patients with small, peripheral semi-solid, or slow-growing lesions may not require a lobectomy (although an anatomic resection is still preferred), ${ }^{6}$ and some studies are indicating favorable long-term outcomes with limited resection. ${ }^{7,8}$ These small ill-defined lesions may be difficult to localize intraoperatively; however, with this technique, they can easily be identified and staged in a directed fashion and in a single 
operative setting. This is important for patients undergoing sublobar resection, as traditional rates of nodal sampling with sublobar resection have been highly variable. ${ }^{11}$ Furthermore, this technique for targeted lymphatic mapping may offer an advantage for poor surgical candidates who cannot tolerate a large anatomic resection but would receive better staging than a patient undergoing focused definitive radiation such as stereotactic body radiation therapy.

In this pilot study, the first patient evaluated was found to have nodal disease solely in the $\mathrm{N} 2$ level $\mathrm{NIR}^{+} \mathrm{SLN}$, which was not detected previously on PET/CT or cervical mediastinoscopy despite multiple LNs sampled including several nodes in the same station. This change in clinical stage significantly changed the prognosis and treatment of the patient resulting in the administration of adjuvant chemotherapy. This disease may have remained occult without the focused direction of the NIR signal as all of the other $\mathrm{NIR}^{-}$nodes in the same nodal station were negative for metastatic disease. Similar upstaging was noted in 2 patients in our prior transpleural NIR trial, reinforcing the importance of targeting specific tumor-associated SLNs for focused pathologic analysis. ${ }^{18}$

Early porcine studies of NB-guided NIR imaging by Anayama and Wada demonstrated the significant potential for clinical translation of a transbronchial ICG injection technique. ${ }^{19,25,26}$ Anayama and colleagues ${ }^{25}$ reported the feasibility of NB-guided ICG delivery adjacent to porcine agar pseudotumors, with NIR signal detection up to a depth of $2.4 \mathrm{~cm}$ and lasting as long as 6 hours in an inflated porcine lung. In the current study, the NIR "tattoo" was readily identifiable in all 12 patients with peritumoral NIR signal detection at depths of up to $3 \mathrm{~cm}$ from the pleural surface. Premixing ICG with HSA, which we have shown previously to be critical for successful SLN identification in humans, also resulted in a stable $\mathrm{NIR}^{+}$signal without significant parenchymal diffusion for more than 4.5 hours after ICG injection. In the study by Wada and colleagues, ${ }^{26}$ attenuated nodal signal was noted with transbronchial ICG injection using ICG concentrations as low as $10 \mu \mathrm{g}$ for lung marking, though previous studies have shown that these low ICG doses are inadequate for human translation of NIR techniques. ${ }^{18,19}$ Thus, using our previously established dose of ICG:HSA, the current trial demonstrates the feasibility of SLN identification after transbronchial injection with persistence of NIR signal in SLN for at least 3 hours after injection, allowing sufficient time for completion of intraoperative diagnosis, resection, and staging.

NB-guided NIR marking and directed surgical resection allows for diagnosis and treatment to be carried out in a single operative setting with the NIR "tattoo" placed via the same NB working channel used for biopsy of a lesion, thus streamlining care, reducing time to therapy, and potentially reducing costs This is in contrast to CT-guided

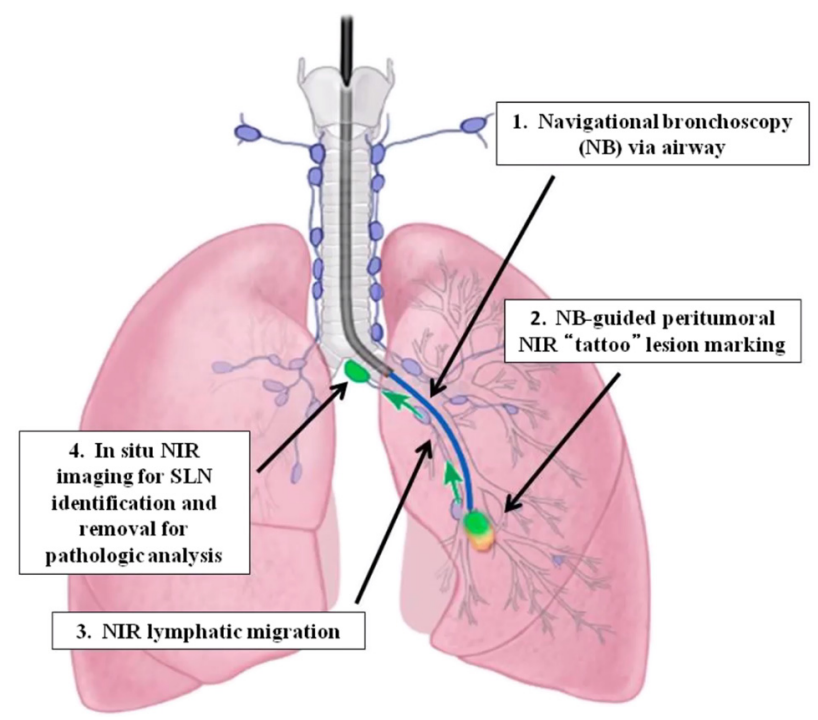

VIDEO 1. A novel technique using navigational bronchoscopy for tumor localization and targeted lymphatic mapping in early-stage lung cancer. Video available at: http://www.jtcvsonline.org/article/S0022-5223(17) 30190-3/addons.

percutaneous biopsy, which typically requires coordination with an interventional radiologist and access to an imaging suite before surgery. In addition, patients are exposed to radiation and are at risk of pneumothorax and possible chest tube placement for the management of complications that may delay definitive diagnosis and treatment. ${ }^{27,28}$ This risk is mitigated with NB as biopsy and ICG injection do not require traversing the pleural surface and may be irrelevant when performed immediately before surgical entry into the chest. The current study demonstrated that NB-guided ICG injection was safe without associated adverse events. Total procedure time including NB-guided peritumoral injection was similar to previous reports adding on average 20 minutes to the operative time for both diagnosis, when indicated, and labeling of the lesions. ${ }^{29}$ Importantly, other approaches to intraoperative detection of pulmonary nodules have been described, including lesion localization with blue dyes, microcoils, percutaneous wire placement, and intravenous ICG, but none of these techniques permit concurrent SLN mapping for the lesion of interest. ${ }^{30-35}$

Blue dyes and radiocolloid tracers have not demonstrated reproducible or accurate SLN detection even in multicenter studies, in large part due to pigmented anthracotic thoracic LNs, high tracer background signal, and rapid migration due to small particle size. ${ }^{36,37}$ The 1 patient in this trial who underwent concurrent methylene blue injection did demonstrate equivalent localization with lesional "tattoo"; however, there was no discernible migration to lymph nodes and the SLN was identified only by ICG. The translational success of other tumor-targeted approaches to fluorescence labeling, such as antibody- 
conjugated and enzyme-activated probes, appears to rely on the ability to predict tumor biology and histology, which can be difficult when evaluating ill-defined lung nodules, particularly when lesions are very small and preoperative histology or genomic sequencing is not available. ${ }^{38,39}$

Limitations of the current prospective pilot trial include a small sample size, limited histologic and radiographic variability in tumor type, and restriction to NB-accessible lesions. Pathologic findings demonstrated the predictive ability of identified $\mathrm{NIR}^{+}$SLNs scrutinized by routine pathologic analysis to identify occult nodal disease, and in the future, in-depth immunohistochemical evaluation may further improve the sensitivity of this approach. In addition, long-term recurrence and survival outcomes are necessary in larger, multicenter trials to determine whether cases identified as NO based on SLN status behave as true node negative cases and exhibit better overall survival than patients deemed node-negative by standard hematoxylin and eosin analysis of routine LAD specimens.

This novel first-in-human prospective pilot trial demonstrates that the NB-guided NIR technique for tumor localization and targeted lymphatic mapping is safe and feasible even for small ill-defined, stage I lung cancers. With continued development, this technology has the potential to significantly extend surgical resection and staging to include nonpalpable lesions in patients that are unable to tolerate a "blind lobectomy" and may alter the way early-stage NSCLC is identified and staged intraoperatively. Under a single anesthetic, patients may have an early-stage NSCLC accurately biopsied for diagnosis, definitively resected, and accurately staged, allowing surgeons to truly achieve the curative benefits purported with early-detection CT-screening programs.

\section{Conflict of Interest Statement}

Authors have nothing to disclose with regard to commercial support.

We acknowledge Novadaq for the donation of ICG dye for the initial 10 patients as proof-of-concept for NB-labeling. The authors would also like to thank Marcia Williams for preparing the illustration used in Figure 1. There has been no preview, restrictions, or direct monetary sponsorship for this trial.

\section{References}

1. American Cancer Society. Cancer Facts \& Figures 2016. Atlanta: American Cancer Society; 2016.

2. Su S, Scott WJ, Allen MS, Darling GE, Decker PA, McKenna RJ, et al. Patterns of survival and recurrence after surgical treatment of early stage non-small cell lung carcinoma in the ACOSOG Z0030 (ALLIANCE) trial. J Thorac Cardiovasc Surg. 2014;2014:747-52.

3. National Lung Screening Trial Research Team, Aberle DR, Adams AM, Berg CD, Black WC, Clapp JD, et al. Reduced lung cancer mortality with low-dose computed tomographic screening. N Engl J Med. 2011;365:395-409.

4. National Lung Screening Trial Research Team, Church TR, Black WC, Berg CD, Clingan KL, et al. Results of initial low-dose computed tomographic screening for lung cancer. N Engl J Med. 2013;368:1980-91.

5. Ma J, Ward EM, Smith R, Jemal A. Annual number of lung cancer deaths potentially avertable by screening in the United States. Cancer. 2013;119:1381-5.

6. Ettinger DS, Wood DE, Akerley W, Bazhenova LA, Borghaei H, Camidge DR, et al. NCCN guidelines insights: non-small cell lung cancer, version 4.2016. J Natl Compr Canc Netw. 2016;14:255-64.

7. Altorki NK, Yip R, Hanaoka T, Bauer T, Aye R, Kohman L, et al. Sublobar resection is equivalent to lobectomy for clinical stage $1 \mathrm{~A}$ lung cancer in solid nodules. J Thorac Cardiovasc Surg. 2014;147:754-62.

8. Schuchert MJ, Kilic A, Pennathur A, Nason KS, Wilson DO, Luketich JD, et al. Oncologic outcomes after surgical resection of subcentimeter non-small cell lung cancer. Ann Thorac Surg. 2011;91:1687-8.

9. Licht PB, Jorgensen OD, Ladegaard L, Jakobsen E. A national study of nodal upstaging after thoracoscopic versus open lobectomy for clinical stage I lung cancer. Ann Thorac Surg. 2013;96:943-9.

10. Medbery RL, Geilespie TW, Liu Y, Nickleach DC, Lipscomb J, Sancheti MS, et al. Nodal upstaging is more common with thoracotomy than with VATS during lobectomy for early-stage lung cancer: an analysis from the national cancer data base. J Thorac Oncol. 2016;11:222-33.

11. Osarogiagbon RU, Yu X. Mediastinal lymph node examination and survival in resected early-stage non-small lung cancer in the surveillance, epidemiology, and end results database. J Thorac Oncol. 2012;7:1798-806.

12. Rusch VW, Hawes D, Decker PA, Martin SE, Abati A, Landreneau RJ, et al Occult metastases in lymph nodes predict survival in resectable non-small-cell lung cancer: report of the ACOSOG Z0040 trial. J Clin Oncol. 2011;29:4313-9.

13. Kubuschok B, Passlick B, Izbicki JR, Thetter O, Pantel K. Disseminated tumor cells in lymph nodes as a determinant for survival in surgically resected nonsmall-cell lung cancer. J Clin Oncol. 1999;17:19-24.

14. Martin LW, D'Cunha J, Wang X, Herzan D, Gu L, Abraham N, et al. Detection of occult micrometastases in patients with clinical stage I non-small-cell lung cancer: a prospective analysis of mature results of CALGB 9761 (Alliance). J Clin Oncol. 2016;34:1484-91.

15. Ludwig MS, Goodman M, Miller DL, Johnstone PA. Postoperative survival and the number of lymph nodes sampled during resection of node-negative non-small cell lung cancer. Chest. 2005;128:1545-50.

16. Osarogiagbon RU, Allen JW, Farooq A, Berry A, O’Brien T. Pathologic lymph node staging practice and stage-predicted survival after resection of lung cancer. Ann Thorac Surg. 2011;91:1486-92.

17. Varlotto JM, Recht A, Nikolov M, Flickinger JC, Decamp MM. Extent of lymphadenectomy and outcome for patients with stage I nonsmall cell lung cancer. Cancer. 2009;115:851-8.

18. Gilmore DM, Khullar OV, Jaklitsch MT, Chirieac LR, Frangioni JV, Colson YL. Identification of metastatic nodal disease in a phase 1 dose-escalation trial of intraoperative sentinel lymph node mapping in non-small cell lung cancer using near-infrared imaging. J Thorac Cardiovasc Surg. 2013;146:562-70.

19. Gilmore DM, Khullar OV, Colson YL. Developing intrathoracic sentinel lymph node mapping with near infrared fluorescent imaging in non-small cell lung cancer. J ThoracCardiovasc Surg. 2012;144:S80-4.

20. Frangioni JV. In vivo near-infrared fluorescence imaging. Curr Opin Chem Biol. 2003; 7:626-34.

21. Schmidt-Hansen M, Baldwin DR, Zamora J. FDG-PET/CT imaging for mediastinal staging in patients potentially resectable non-small cell lung cancer. JAMA 2015;313:1465-6.

22. Cho H, Lee HY, Kim J, Kim HK, Choi JY, Um SW, et al. Pure ground glas nodular adenocarcinomas: are preoperative positron emission tomography/ computed tomography and brain magnetic resonance imaging useful or necessary? J Thorac Cardiovasc Surg. 2015;150:514-20.

23. Darling GE, Allen MS, Decker PA, Ballman K, Malthaner RA, Inculet RI, et al. Randomized trial of mediastinal lymph node sampling versus complete lymphadenectomy during pulmonary resection in the patient with N0 or N1 (less than hilar) non-small cell carcinoma: results of the American College of Surgery Oncology Group Z0030 trial. J Thorac Cardiovasc Surg. 2011; 141:662-70.

24. Boyle JM, Tandberg DJ, Chino JP, D'Amico TA, Ready NE, Kelsey CR. Smoking history predicts for increased risk of second primary lung cancer: a comprehensive analysis. Cancer. 2015;121:598-604.

25. Anayama T, Qiu J, Chan H, Nakajima T, Weersink R, Daly M, et al. Localization of pulmonary nodules using navigation bronchoscope and a near-infrared fluorescence thoracoscopy. Ann Thorac Surg. 2015;99:224-30.

26. Wada H, Hirohashi K, Anayama T, Nakajima T, Kato T, Chan HH, et al Minimally invasive electro-magnetic navigational bronchoscopy-integrated 
near infrared-guided sentinel lymph node mapping in the porcine lung. PLoS One. 2015;10:e0126945.

27. Dale CR, Madtes DK, Fan VS, Gorden JA, Veenstra DL. Navigational bronchoscopy with biopsy versus CT-guided biopsy for the diagnosis of a solitary pulmonary nodule: a cost-consequences analysis. J Bronchology Interv Pulmonol. 2012;19:294-303.

28. Wang Memoli JS, Neitert PJ, Silvestri GA. Meta-analysis of guided bronchoscopy for the evaluation of the pulmonary nodule. Chest. 2012;142:385-93.

29. Eberhardt R, Anantham D, Herth F, Feller-Kopman D, Ernst A. Electromagnetic navigation diagnostic bronchoscopy in peripheral lung lesions. Chest. 2007;131: 1800-5.

30. Krimsky WS, Minnich DJ, Cattaneo SM, Sarkar SA, Harley DP, Finley, et al Thoracoscopic detection of occult indeterminate pulmonary nodule using bronchoscopic pleural dye marking. J Community Hospital Intern Med Perspect. 2014;4:23084-9.

31. Galetta D, Bellomi M, Grana C, Spaggiari L. Radio-guided localization and resection of small or ill-defined pulmonary lesions. Ann Thorac Surg. 2015; 100:1175-80.

32. Finley RJ, Mayo JR, Grant K, Clifton JC, English J, Leo J, et al. Preoperative computed tomography-guided microcoil localization of small peripheral pulmonary nodules: a prospective randomized controlled trial. J Thorac Cardiovasc Surg. 2015;149:26-32.

33. Chen S, Zhou J, Zhang J, Hu H, Luo X, Zhang Y, et al. Video-assisted thoracoscopic solitary pulmonary nodule resection after CT-guided hookwire localization: 43 cases report and literature review. Surg Endosc. 2011;25:1723-9.
34. Okusanya OT, Holt D, Heitjan D, Deshpande C, Venegas O, Jiang J, et al. Intraoperative near-infrared imaging can identify pulmonary nodules. Ann Thorac Surg. 2014;98:1223-30.

35. Rosenthal EL, Warram JM, de Boer E, Basilion JP, Biel MA, Bogyo M, et al. Successful translation of fluorescence navigation during oncologic surgery: a consensus report. J Nucl Med. 2016;57:144-50.

36. Liptay MJ, D’Amico TA, Nwogu C, Demmy TL, Wang XF, Gu L, et al. Intraoperative sentinel node mapping with technitium-99 in lung cancer: results of CALGB 140203 multicenter phase II trial. J Thorac Oncol. 2009; 4:198-202.

37. Rzyman W, Hagen OM, Dziadziuszko R, Kobierska-Gulida G, Karmolinski A, Lothe MI, et al. Blue-dye intraoperative sentinel lymph node mapping in early non-small cell lung cancer. Eur J Surg Oncol. 2006;32:462-5.

38. Nguyen QT, Olson ES, Aguilera TA, Jiang T, Scadeng M, Ellies LG, Tsien RY. Surgery with molecular fluorescence imaging using activatable cell-penetrating peptides decreases residual cancer and improves survival. Proc Natl Acad Sci USA. 2010;107:4317-22.

39. Strong VE, Humm J, Russo P, Jungbluth A, Wong WD, Daghighian F, et al. A novel method to localize antibody-targeted cancer deposits intraoperatively using handheld PET beta and gamma probes. Surg Endosc. 2008;22: 386-91.

Key Words: near-infrared, navigational bronchoscopy, tumor localization, sentinel lymph node, lung cancer 